\title{
Reflections on some aspects of ground-based observations for asteroseismology
}

\author{
Christiaan Sterken \\ Vrije Universiteit Brussel, Pleinlaan 2, B-1050 Brussels, Belgium
}

\begin{abstract}
We call attention to two problems of long-term observations: the problem of maintaining reliable and stable standardization, and the problem of securing sufficient power in terms of postdoctoral workers to carry out the workload.
\end{abstract}

\section{Introduction}

This meeting clearly illustrates that there is taking place a happy evolution in the study of pulsating stars, notably the gradual increase in the length of the time baseline of observations. In particular, the old habit to "observe many stars just a little bit" is gradually giving way to monitoring a more limited number of objects over much longer time baselines. The growing opinion that good science comes from long strings of data is a very positive development. That this has not always been the case, is testified by a statement from Paul Ledoux ${ }^{1}$ :

"... I do not want to deny the importance of statistical relations that might be revealed by numerous but limited observations of a great number of objects. But it seems to me that real progress in the physical interpretation of a given type of variables depends essentially on detailed and at the same time complete and continuous observations of one typical star."

Indeed, many projects now cover an unprecedented extent in time coverage, and in precision. At the same time, surveys almost double the number of known members of a class of variables from one major meeting to the other.

Wojtek Dziembowski underlined that mode identification relies on theory, and not only on observational data. Indeed, there is a long distance between delivering a complete and accurate frequency solution, and recognizing modes: much more is needed than good data and a frequency solution. Pulsation-mode identification heavily relies on sophisticated theories, and the increasing computational facilities soon will force us to deal with millions of models and tracks. But these complicated models are by far not the only theoretical aspect of asteroseismology: few observers realize that there is a tremendous impact of theoretical conceptions on so-called "observables", i.e. quantities that are not observed directly, but always depend on theory, mainly through their calibration.

The foregoing thoughts lead to the recognition of two problems.

\section{Problem I}

The bonus of extending the observational baseline has a drawback, though. It is not often realized that long time bases frequently lead to problems of standardization. Let me remind that standards are not just a set of constant stars needed for transforming one batch of data from one site to another: standards are a system of basic calibrators needed to guarantee

\footnotetext{
${ }^{1} 1956$, letter to C. de Jager.
} 
the stability and consistency of the observational data, and to secure a reliable and stable (in time) mapping of the space of basic data (e.g. colour indices) onto the space of physical "observables" (e.g. $T_{\text {eff }},[\mathrm{Fe} / \mathrm{H}]$, or even angular diameter).

We must realize that existing standards and calibrations change with time, mainly because detectors change. Unfortunately, most of this is poorly documented. Moreover, almost every new observing facility (ground- and space based) creates a new standard that is not compatible with basic calibrations of stellar observables constructed a decade or longer ago. Last but not least, the acquisition of calibrated measurement is poorly taught during observer training.

\section{Problem II}

Referring to the shiny prospects ahead, someone said "We should all be in business for an extended period of time to come." Yes, but. Have a look at the mode of funding of so many projects presented here: funding agencies provide huge amounts of support to acquire the data, money for organizing meetings, and travel support to populate these meetings. However, they do not provide long-term or even permanent research positions to guarantee that we shall ever be able to fully analyse and truly understand the new data flowing in. It is time to change these modes of funding, and perhaps we should use the momentum of these new networks to convince governments and funding agencies to invest more means in salaries for postdoctoral researchers.

\section{DISCUSSION}

Roxburgh: I agree with you that we should change the way of funding. But how?

Sterken: By just trying. You have to insist, and I think that people like you, Michel and others are in a position to revert this tendency. As a postdoc, it is nice to travel etc., but it is not so nice when after a few years, when you understand what you are doing and you like what you are doing, you will have to stop.

Metcalfe: I would like to point out that governments and funding agencies are not the only people to whom we should make these arguments. Two examples already exist: the Delaware Asteroseismic Research Center (which now operates the Whole Earth Telescope), and the Las Cumbres Observatory Global Telescope Network (also known as the Google network), are both privately funded.

Shipman: There is actually a third example in the US that we are sometimes taking advantage of. This is the SMARTS organization which is running the smaller telescopes at Cerro Tololo in Chile. In part, these smaller telescopes networks, in particular the SONG project, are really trying to reduce operating costs. Another comment from my observations in the States is that when I am trying to make implicit longer-term commitments, I never mentioned the word tenure in connection with supporting the people associated with the project. I know that if I had mentioned that, I would have had no success at all in getting any money.

Deupree: You need to appreciate the amount of time it takes senior people (or anybody else) to work for these sorts of changes. It takes many visits to the appropriate people to lobby for these changes - they cannot be made to happen quickly. With luck these types of changes can be made, but it usually requires significant sacrifices of time by senior personnel to make it happen.

Sterken: That is true, but you need to invest a lot of time on grant applications anyway. If you are doing a long-term project and the funding stops after a few years, all this is lost. I agree that you must spend time on it, but only once to make this suggested change. 